\title{
Testing measurement invariance and latent mean differences across gender groups in college students' Internet-specific epistemic beliefs
}

\author{
Yen-Lin Chiu, Chin-Chung Tsai, Jyh-Chong Liang \\ National Taiwan University of Science and Technology, Taiwan
}

\begin{abstract}
The purposes of this study were to investigate the measurement invariance and gender differences in the Internet-specific epistemic beliefs between male and female undergraduates. A total of 735 university students in Taiwan were surveyed using the Internet-specific epistemic beliefs questionnaire (ISEQ). By conducting structural equation modeling (SEM), the measurement invariance and latent mean comparisons across gendered groups were tested. After the invariance tests were all satisfied, the latent mean analysis approach was conducted. The results of the latent mean comparisons revealed that a gender gap occurred in the uncertainty, complexity and source of Internet-specific epistemic beliefs; however no gender difference was found in the justification dimension. In general, the study findings suggest that differences in beliefs regarding Internet-based knowledge exist between undergraduate males and females. The gendered issue of Internet-based learning and epistemic beliefs cannot be ignored. Finally, some suggestions for developing Internetrelated curricula and instruction were also proposed.
\end{abstract}

\section{Introduction}

As its accessibility and convenience improves, the Internet has become the major resource of information for students working on academic tasks or course assignments. Since college students rely heavily on the Internet for academic information, their abilities of evaluating the credibility of online information sources have become an issue of concern (Biddix, Chung, \& Park, 2011; Liang \& Tsai, 2009; Metzger, Flanagin, \& Zwarun, 2003). In the Internet context, students have to evaluate and justify the mass of information they retrieve from websites on their own; therefore there is a need to activate their epistemic judgment while searching for online information (Bråten, Strømsø, \& Samuelstuen, 2005; Mason \& Boldrin, 2008). The role of epistemic beliefs in learners' Internet-based learning activities has been investigated (Bråten \& Strømsø, 2006; Tsai, 2008). In particular, the influences of Internet-specific epistemic beliefs (i.e., perceptions regarding the nature of Internet-based knowledge and knowing) on learning in Internet-based environments also have been examined (Bråten et al., 2005; Chiu, Liang, \& Tsai, 2013).

With increasing utilisation of information technology in economic and academic arenas, digital divide that differs with genders remains a social problem which should be concerned (Cooper, 2006). Selwyn (2007) claimed that the stereotype of educational computing as a masculine activity may prohibit females from learning with new technologies. Although female students tend to regard the Internet as a useful tool for study, they are more likely than males to underestimate their competence in using the Internet for learning (Bråten \& Strømsø, 2006; Huang, Hood, \& Yoo, 2013; Peng, Tsai, \& Wu, 2006). On the other hand, male students reveal more confidence to use the Internet than females do; however they tend to perceive the Internet as toy rather than tool for study (Peng et al., 2006). Within the Internet-based learning environment, the issue of a gender-related digital divide was extensively explored and debated (Selwyn, 2007; Tsai, Liang, Hou, \& Tsai, 2012; Tsai \& Tsai, 2010). Instead of access to the Internet, intention toward and perceptions of the Internet-based learning become the major issues of gender-related digital divide (Chen \& Tsai, 2007; Huang et al., 2013).

A better understanding of students' perceptions of Internet-based learning may assist instructors in developing appropriate curricula and learning environments for both male and female students (Chuang, Hwang, \& Tsai, 2008; Gonzalez-Gomez, Guardiola, Rodriguez, \& Alonso, 2012; Selwyn, 2007). The influential role of Internet-specific epistemic beliefs in students' Internet-based learning has been explored (Bråten et al., 2005; Chiu et al., 2013; Strømsø \& Bråten, 2010), however the issue of gender differences in Internet-specific epistemic beliefs has not yet been discussed in the online learning context. This study aimed to examine whether there is a gap of Internet-specific epistemic beliefs between male and female 
undergraduates while searching their course-related information online. Rather than the traditional analysis techniques, the latent mean analyses with structural equation modeling were conducted to test such genderrelated digital divide.

\section{Literature review}

\section{The role of epistemic beliefs in Internet-based learning}

Personal epistemology can be defined as individual's beliefs about the nature of knowledge concerning what knowledge is and the nature of knowing relating to how one comes to know (Hofer \& Pintrich, 1997). Researchers have concluded learners' perceptions of knowledge and knowing (i.e., epistemic beliefs) may have influential effects on their learning motivation and their approaches to processing information (Hofer \& Pintrich, 1997; Schommer, 1990). In Internet-based learning environments, it has been indicated that students' epistemic beliefs are related to their preference for such learning contexts (Tsai \& Chuang, 2005). Students with more sophisticated epistemic beliefs have a greater preference for web-based learning environments than those with naïve beliefs (Yang \& Chang, 2009). Because of the abundant nature of Internet-based information, students' epistemic beliefs may act as an essential tool determining their judgment related to the validity of the information on the Internet (Tsai, 2004). There have also been several studies considering the role of epistemic beliefs in Internet-based learning environments (Bråten \& Strømsø, 2006; Mason, Ariasi, \& Boldrin, 2011; Tsai, 2008). For instance, it has been indicated that students' epistemic beliefs may predict their Internet-search activities such as Internet-based discussion and communication activities (Bråten \& Strømsø, 2006).

\section{The role of Internet-specific epistemic beliefs in Internet-based learning}

Since the Internet gives presentation of knowledge and process of knowing in new ways, it has been claimed that measures of epistemic beliefs should focus on the nature of knowledge and knowing in Internet-specific environments (Bråten et al., 2005). As the context-sensitivity of epistemic beliefs has been emphasized, there is a need to investigate students' epistemic beliefs, specifically in the Internet context (Mason \& Boldrin, 2008; Mason et al., 2011). After constructing the Internet-specific epistemic beliefs, Bråten and his colleagues (2005) indicated that students' Internet-specific epistemic beliefs may predict their learning activities, including Internet search and Internet communication. Strømsø and Bråten (2010) also found that students' Internet-specific epistemic beliefs are related to their self-regulatory strategies of Internet-based learning. Moreover, it was revealed that Internet-specific beliefs may be linked to self-regulated learning process while searching academic information online (Chiu et al., 2013).

\section{Gender differences in Internet use and Internet-based learning}

With respect to the advantages of the Internet and its vast integration into education, the digital divide in gender has been intensively concerned in the Internet-based learning environment (e.g., Chuang et al., 2008; Huang et al., 2013; Tsai et al., 2012). Digital divide referring to various inequalities in access to, and use of, technology may be conceived as a serious social problem, since some individuals may utilise the advantages of technology while others will not (Jones, Johnson-Yale, Millermaier, \& Perez, 2009). The gender-related digital divide, one type of gendered differences in technology use, is a critical issue and has been discussed with regard to the use of information technology and computers in education (Cooper, 2006; Volman \& van Eck, 2001; Wong, Teo, \& Russo, 2012). Although the latest generation of learners has grown up in a technology-saturated environment, they still represent gender stereotyping perceptions across different computing applications (Selwyn, 2007). The gender stereotype that computers and the Internet are masculine technologies may result in the gender differences in students' self-efficacy and attitudes toward their utilisation of the Internet and the Internet-based learning (Jones et al., 2009; Selwyn, 2007; Sherman et al., 2000), therefore exploring the gender differences in Internet-based learning is important for educators to encourage students to participate in such learning (Chuang et al., 2008; Huang et al., 2013).

In addition to the gendered issue of computer use, gender differences in use of, and attitudes toward, the Internet have also been broadly explored (Chou, Wu, \& Chen, 2011; Jones et al., 2009; Sherman et al., 2000), however there are still debates and conflicting findings on the issue of gender differences in Internet use. A survey on the use of the Internet has shown that there is no gender difference in Internet access; females use the Internet as frequently as males (Wasserman \& Richmond-Abbott, 2005). It has been claimed 
that the gender divide in access to the Internet has narrowed (Dholakia, 2006). Shaw and Gant (2002) found no gender differences in Internet use for interpersonal communication, and concluded that the gender gap is rapidly narrowing among the younger generation.

On the contrary, numerous other researchers have recognised a gender gap in Internet use or in attitudes towards it. Sherman et al. (2000) claimed that gender differences in undertaking Internet-related activities have not diminished among college students. Even though access to computing devices and Internet services has increased, it has been indicated that the digital divide will not cease to exist (Jones et al., 2009). One empirical study found that gender differences exist in university students' attitudes toward and perceptions of the Internet; the perceived control over the Internet possessed by male students is higher than that of female students (Peng et al., 2006). Gender differences also occur in the types of Internetrelated activities undertaken, with males tending to partake in leisure and communication activities on the Internet more often than females (Chou et al., 2011; Li \& Kirkup, 2007).

For Internet-based learning, differences between male and female students have been found in their perceptions of Internet-based learning environments, with male students displaying better adaptability than female students to the Internet-based learning approach (Chuang et al., 2008). Males and females perceive online learning differently, with males reporting higher ratings of perceived usefulness, perceived ease of use, and behavioural intention toward such learning than females (Ong \& Lai, 2006). However in a selfregulated online learning environment, gender differences were not found in terms of motivational beliefs, self-regulated learning variables, or achievement in programming (Yukselturk \& Bulut, 2009). Although females may regard the Internet as a masculine technology, female university students tend to possess more positive attitudes than male students toward the helpfulness and varied content of web-based learning (Chen \& Tsai, 2007). Contrary to the smaller number of male college students, a large number of female college students report that their most frequent use of the Internet is for academic work (Jones et al., 2009). Compared with male university students, female students express greater satisfaction with their online learning subjects (Gonzalez-Gomez et al., 2012). It has been claimed that studying online can be seen as a feminine activity (Selwyn, 2007).

In sum, the gender differences among Internet use and Internet-based learning are reported with mixed findings. In particular, this study further examined the gender differences in college students' Internetspecific epistemic beliefs underlying Internet-based learning processes.

\section{Gender differences in epistemic beliefs}

Reviewing previous research on general epistemic beliefs, evidence for gender differences within such constructs remains mixed (Kessels, 2013). Moreover, Conley, Pintrich, Vekiri, and Harrison (2004) found no evidence for either main or moderating effects of gender on students' development of domain-specific epistemic beliefs. Nor were there significant differences in epistemic reasoning level between male and female university students (Kessels, 2013). Neither was a significant gender difference found in university students' epistemic beliefs related to science (Rizk, Jaber, Halwany, \& BouJaoude, 2012). However gender difference in epistemic beliefs showed that men hold stronger beliefs regarding the certainty of knowledge than women (Bendixen, Schraw, \& Dunkle, 1998). In Tsai’s (2006) study, it was reported that male and female students demonstrate different beliefs related to their science epistemic views; male students tend to have more mature epistemic beliefs related to physics and biology than do female students. Gender has been regarded as an influential factor of student's scientific epistemic views (Chai, Deng, \& Tsai, 2012).

According to the aforementioned studies, there are no consistent findings regarding the gender difference issue in students' epistemic beliefs. In addition, in these studies the epistemic beliefs examined were mainly general epistemic thinking rather than in specific domains or contexts (e.g., Bendixen et al., 1998; Conley et al., 2004), except for several studies focused on specific domains such as scientific knowledge (e.g., Chai et al., 2012; Rizk et al., 2012), as well as biological and physics knowledge (e.g., Tsai, 2006). In Internetbased learning environments, epistemic beliefs and gender-related issues have been issues of concern and have been used to predict students’ Internet-based learning activities (Bråten \& Strømsø, 2006) and preference for Internet-based learning environments (Tsai, 2008). Bråten and Strømsø (2006) have indicated that rather than the Internet-based environment, the characteristics of learners such as epistemic beliefs and gender determine whether the Internet-based environment may deliver good learning outcomes for learners. In particular, students’ Internet-specific epistemic beliefs may be linked to their Internet-based 
learning activities (Bråten et al., 2005; Chiu et al., 2013; Strømsø \& Bråten, 2010). However since the gender differences in epistemic beliefs regarding Internet-based learning activities has not yet been carefully examined, further studies should especially focus this gender issue on Internet-specific epistemic beliefs. Identifying gender differences in Internet-specific epistemic beliefs can better understand that male and female students could have potentially different outcomes in Internet-based learning environments. The findings of such gender-related issue may provide insights for instructors to better administer Internet-based learning activities.

\section{Measurement invariance and latent mean analysis}

In previous studies most of the gender comparisons of Internet-related activities were conducted utilising t-tests (e.g., Li \& Kirkup, 2007; Peng et al., 2006). Rather than the traditional approaches such as $t$-test and analysis of variance (ANOVA), the latent mean analysis approach has been recommended to be used to undertake comparisons across groups while the variables of interest are conceptualised as latent constructs that cannot be directly measured (Hong, Malik, \& Lee, 2003; Kuhn \& Holling, 2009). Since comparative investigations are often conducted using quantitative methodology, it may raise concerns about whether certain instruments give a valid basis for group comparisons (Gregorich, 2006). Before comparing the latent mean differences across groups, measurement invariance is an essential prerequisite (Chen, 2007; Cheung \& Rensvold, 2002). Measurement invariance tests can determine whether instrument items mean the same thing to subjects of different groups (Cheung \& Rensvold, 2002). To compare the gender differences in Internet-specific epistemic beliefs, measurement invariance tests and latent mean analysis were executed in this study.

\section{Method}

\section{Participants}

The participants of this study were 735 undergraduates from seven universities in Taiwan; 389 males and 346 females. Seven teachers in those universities were requested to provide assistance in delivering the questionnaire. These teachers contacted their students by sending email to a potential list, and invited them to answer the questionnaire online. All the participants were voluntary respondents and satisfied the recruiting criteria of having experience in seeking online academic information for their course-related assignments. Their personal information and responses were promised to be confidentially treated. The domains of these participants were engineering, science, social science, humanities, education as well as medicine. Their average age was $21.13(S D=1.54)$. In sum, 85.4\% reported using the Internet for more than 10 hours per week. Most (87.9\%) of the respondents also reported that they used the Internet for academic information searching at least once a week.

\section{Measures}

While conducting this survey, all participants responded to the measures by assessing their perceptions of academic information searching for course assignments on the Internet. Hofer and Pintrich (1997) refered to personal epistemology as beliefs consist of independent dimensions regarding knowledge and knowing. According to Hofer and Pintrich's (1997) theory of personal epistemology, Bråten et al. (2005) drew a four dimension theoretic construct of Internet-specific epistemic beliefs concerning knowledge and knowing in Internet-based environments. The Internet-Specific Epistemic Questionnaire (ISEQ) developed by Bråten and his colleague (2005) has been translated into Chinese and validated by Chiu et al. (2013) on a sample of university students. In accordance with Hofer and Pintrich's (1997) theory, the Chinese version of ISEQ was constructed as a four dimension model, consisting two dimensions relating to Internet-based knowledge, namely certainty of Internet-based knowledge and simplicity of Internet-based knowledge, as well as two dimensions regarding Internet-based knowing, namely source of Internet-based knowledge and justification for Internet-based knowing. Except for the source of Internet-based knowledge and justification for Internet-based knowing, to better comprehend the constructs of ISEQ in this study, the remaining two dimensions were relabeled as uncertainty of Internet-based knowledge and complexity of Internet-based knowledge respectively. The four dimensions, containing respectively three items regarding Internet-based knowledge and knowing, were evaluated. Higher scores represented more sophisticated Internet-specific epistemic beliefs. 
Uncertainty of Internet-based knowledge measured the belief that the knowledge on the Internet is uncertain, tentative and changeable rather than certain, true and accurate. High scores on uncertainty indicated that respondents were more likely to doubt the certainty of course-related information found on the Internet. Sample item: The Internet contains correct answers related to questions about my course work (reversed item).

Complexity of Internet-based knowledge evaluated the belief that the knowledge located on the Internet is complex and interrelated concepts rather than simple and accumulations of specific details. Students with high scores on complexity tended to view the course-related knowledge on the Internet as complex concepts. Sample item: The Internet offers abundant details about topics related to my study (reversed item).

Source of Internet-based knowledge assessed the belief that the Internet is not a definite source originating and transmitting completed and correct knowledge. Higher scores on source represented that respondents were inclined to doubt that the Internet is a good source offering essential and correct information about their study. Sample item: Various sources on the Internet provide the correct answer to questions related to my course work (reversed item).

Justification for Internet-based knowing assessed the belief that the knowledge on the Internet should be evaluated and reasoned. High scores on justification indicated respondents' views that knowledge claims on the Internet should be checked with diverse sources. Sample item: I would evaluate the logicality of the course-related knowledge that I find on the Internet.

\section{Statistical analyses procedures}

The approach of latent mean analysis (Hong et al., 2003) was used to compare the gender differences with respect to college students' Internet-specific epistemic beliefs. To conduct the latent mean analysis, structural equation modeling (SEM) was performed using AMOS 18.0. Measurement invariance is the prerequisite for comparing the differences across groups with the latent means analysis approach (Chen, 2007; Hong et al., 2003; Kuhn \& Holling, 2009). Measurement invariance concerns whether the measure items and structural factors have the same meanings across groups (Kuhn \& Holling, 2009). An examination of measurement invariance can reveal whether the instrumentation is a valid basis for comparison of group differences (Gregorich, 2006). Before testing the group differences in the means of latent variables, the configural invariance, metric invariance, and scalar invariance must be examined to evaluate the equivalence of the groups (Hong et al., 2003; Jang, Lee, Puig, \& Lee, 2012).

The measurement invariance tests are a series of hierarchically nested confirmatory factor analyses by increasing levels of cross-group equality constraints (Gregorich, 2006; Kuhn \& Holling, 2009). First, configural invariance tests whether each common factor is associated with identical items across groups (Gregorich, 2006), fixing construct dimensionality to be invariant (Kuhn \& Holling, 2009). The second level of invariance, namely metric invariance, is tested by constraining the factor loadings to be equivalent across groups (Hong et al., 2003; Jang et al., 2012). Third, scalar invariance is tested at the intercept level, whereby the intercepts are constrained to be equal across groups (Jang et al., 2012), to examine whether the comparisons of group means are meaningful (Gregorich, 2006). Usually, the fourth invariance, called strict measurement invariance, is to assess the residual equivalence across groups (Gregorich, 2006; Kuhn \& Holling, 2009), however due to a variety of reasons, residual invariance is difficult to satisfy (Chen, 2007). Residual invariance is not necessary for meaningful comparisons of group means, and itself is of limited practical value (Gregorich, 2006).

To determine how well the sample data fit a proposed model, the cutoff values of model fit indices recommended by Byrne (2010) and Kline (2005) were employed as criteria. The ratio of chi-square to degrees of freedom less than $5\left(\chi^{2} / d f<5\right)$ indicates acceptable fit. The root mean square error of approximation (RMSEA) value less than 0.05 indicate good fit. The comparative fit index (CFI) larger than 0.9 is considered a well model fit. With respect to the invariance tests, the non-significant result of the chisquare difference test and the change value of CFI smaller than 0.01 indicate that the invariance tests claimed are supported (Byrne, 2010; Cheung \& Rensvold, 2002, Chen, 2007). 


\section{Results}

\section{Descriptive statistics}

The male and female students' means and standard deviations for the 12 items of ISEQ were respectively presented in Table 1. To examine the normality of each item, the skewness and kurtosis values were also displayed in Table 1. As shown, the values of univariate skewness and kurtosis for the males and females range from -1.27 to 2.78. As recommended by Byrne (2010) and Bandalos and Finney (2010), an absolute value of 7 was used as a guide. All values are greater than -7 and less than 7 , indicating that no item is considered to have a severe normality problem. However, the Mardia's coefficients of multivariate kurtosis in the male and female samples are 37.66 and 29.47 respectively, showing a problem of multivariate nonnormality. According to Byrne's (2010) recommendation, if the Mardia's coefficient has a value greater than 5 , then bootstrapping should be conducted. Therefore, bootstrapping was used in this study to analyse the estimates.

Table 1

Means, standard deviations and normality tests for male and female students

\begin{tabular}{ccccccccc}
\hline & \multicolumn{3}{c}{ Male students $(n=389)$} & \multicolumn{3}{c}{ Female students $(n=346)$} \\
\hline Items & $M$ & $S D$ & skewness & kurtosis & $M$ & $S D$ & skewness & kurtosis \\
\hline UCE1 & 3.87 & 1.42 & 0.34 & -0.49 & 4.21 & 1.30 & 0.08 & -0.61 \\
UCE2 & 3.61 & 1.39 & -1.27 & 2.78 & 3.92 & 1.28 & -0.46 & 0.05 \\
UCE3 & 3.21 & 1.33 & -0.98 & 2.25 & 3.50 & 1.33 & -0.47 & 0.25 \\
CP1 & 3.25 & 1.29 & -0.95 & 1.88 & 3.60 & 1.31 & -0.45 & 0.11 \\
CP2 & 3.16 & 1.19 & 0.36 & -0.33 & 3.45 & 1.17 & 0.21 & -0.45 \\
CP3 & 3.19 & 1.20 & 0.53 & -0.09 & 3.43 & 1.20 & 0.42 & -0.23 \\
SO1 & 3.43 & 1.40 & 0.75 & 0.50 & 3.72 & 1.35 & 0.33 & -0.28 \\
SO2 & 3.52 & 1.44 & 0.70 & 0.64 & 3.77 & 1.44 & 0.35 & -0.23 \\
SO3 & 3.74 & 1.49 & 0.59 & 0.75 & 4.03 & 1.42 & 0.23 & -0.08 \\
JU1 & 5.66 & 1.11 & 0.77 & 0.93 & 5.68 & 0.96 & 0.60 & 0.22 \\
JU2 & 5.75 & 1.01 & 0.51 & -0.20 & 5.69 & 0.88 & 0.12 & -0.75 \\
JU3 & 5.67 & 1.03 & 0.54 & -0.25 & 5.66 & 0.92 & 0.35 & -0.41 \\
Multivatiate & & & & 37.66 & & & & 29.47 \\
\hline
\end{tabular}

\section{Measurement invariance}

Before undertaking the measurement invariance tests, the confirmatory factor analyses were separately conducted for the male and female groups. The analyses may reveal whether ISEQ can represent the same number of common factors across divergent groups (Gregorich, 2006). According to Chiu et al.'s (2013) tests of reliability and validation, in this study the ISEQ was also identified as an Internet-based epistemic model with four dimensions. Due to the problem of multivariate non-normality, bootstrapping was used to conduct the confirmatory factor analyses for the male and female students respectively. As shown in Table 2a and Table $2 b$, factor loadings range from 0.69 to 0.91 for the male students, and from 0.62 to 0.88 for the female students. In addition, the factor loadings with $90 \%$ confidence interval were presented. Model fit indices for both male and female students were also displayed in the notes of Table 2a and Table $2 \mathrm{~b}$. Based on the criteria recommended by Byrne (2010) and Kline (2005), the model fit indices for both groups suggest a good data-model fit (i.e., $\chi^{2} / d f<5$, RMSEA $<0.05$, CFI $>0.90$ ). 
Table 2a

Factor loadings of CFA and Cronbach's $\alpha$ for ISEQ by males

\begin{tabular}{|c|c|c|c|c|}
\hline \multicolumn{5}{|c|}{ Male students $(n=389)$} \\
\hline Items & UCE & $\mathrm{CP}$ & SO & $\mathrm{JU}$ \\
\hline UCE1 & $0.81(0.76,0.85)$ & & & \\
\hline UCE2 & $0.83(0.78,0.87)$ & & & \\
\hline UCE3 & $0.69(0.62,0.75)$ & & & \\
\hline CP1 & & (0.75, & & \\
\hline $\mathrm{CP} 2$ & & (0.66, & & \\
\hline CP3 & & (0.79, & & \\
\hline SO1 & & & (0.66, & \\
\hline $\mathrm{SO} 2$ & & & $(0.78$ & \\
\hline SO3 & & & (0.89, & \\
\hline JU1 & & & & $0.87(0.80,0.91)$ \\
\hline JU2 & & & & $0.79(0.73,0.85)$ \\
\hline JU3 & & & & $0.83(0.76,0.88)$ \\
\hline Cronbach's $\alpha$ & 0.82 & 0.83 & 0.86 & 0.87 \\
\hline
\end{tabular}

Notes: Factor loadings are standardised values. Values of Cronbach's $\alpha$ are in boldface.

Factor loadings with $90 \%$ confidence interval are written in parentheses.

Model fit indices: $\chi^{2}=88.154 ; d f=48 ; \chi^{2} / d f=1.837$; GFI $=0.964$; AGFI $=0.942 ;$ CFI $=0.984$; $\mathrm{RMSEA}=0.046$.

UCE: uncertainty; CP: complexity; SO: source; JU: justification.

Table $2 \mathrm{~b}$

Factor loadings of CFA and Cronbach's $\alpha$ for ISEQ by females

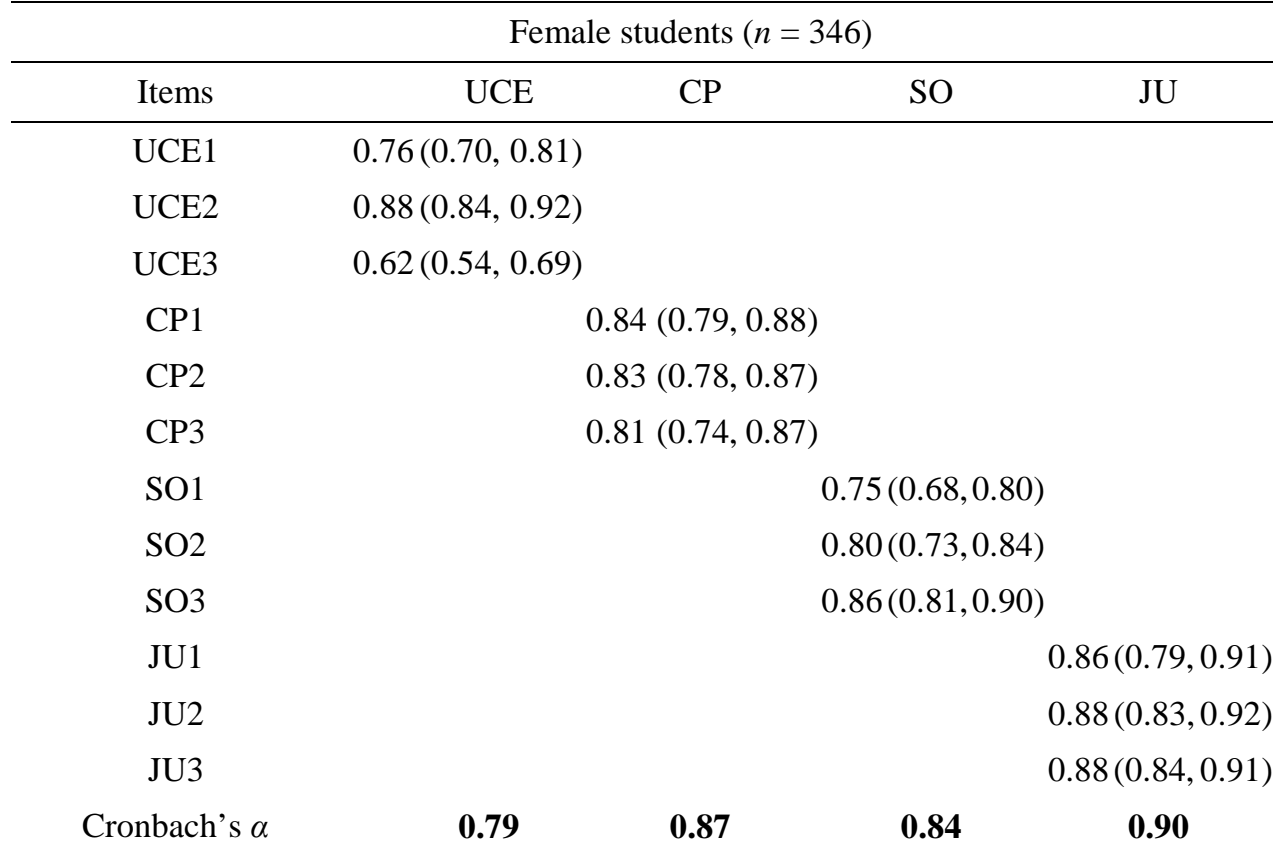

Notes: Factor loadings are standardised values. Values of Cronbach's $\alpha$ are in boldface.

Factor loadings with $90 \%$ confidence interval are written in parentheses.

Model fit indices: $\chi^{2}=82.531 ; d f=48 ; \chi^{2} / d f=1.719$; GFI $=0.963$; AGFI $=0.939$; CFI $=0.985 ;$ RMSEA $=0.046$.

UCE: uncertainty; CP: complexity; SO: source; JU: justification. 
Before conducting latent mean analysis, several invariance tests were performed. First, multi-group SEM was executed to assess configural invariance by analysing the two groups without constraining equality across the groups. As shown in Table 3, the results of goodness-of-fit $\left(\chi^{2}=170.684, d f=96, \chi^{2} / d f=1.778\right.$, CFI $=0.985$, RMSEA $=0.033$ ) in the configural invariance test indicate that the structural patterns are similar across groups. This implies that the configural model can be a baseline to compare with other restricted models in the invariance hierarchy. Second, metric invariance was performed by constraining the factor loadings to be equal across groups. The results of the metric invariance model $\left(\chi^{2}=178.914, d f=\right.$ $104, \chi^{2} / d f=1.72$, CFI $=0.985$, RMSEA $=0.031$ ) presented in Table 3 suggest a well model fit. The nonsignificant result of the chi-square difference test indicates that metric invariance is claimed (Byrne, 2010). According to the recommended criteria (Cheung \& Rensvold, 2002), a change value of CFI $(\triangle \mathrm{CFI}=0$ ) smaller than 0.01 also gives evidence to support the metric invariance hypothesis. Third, a scalar invariance test was performed by restricting the intercepts across groups to be invariant. In Table 3, the model fit indices of the scalar invariance model $\left(\chi^{2}=201.768, d f=116, \chi^{2} / d f=1.739, \mathrm{CFI}=0.982\right.$, RMSEA $\left.=0.032\right)$ were displayed. Although, the chi-square difference between the metric model and the scalar model is significant $(p<0.05)$, the change value of CFI $(\triangle \mathrm{CFI}=0.003)$ is smaller than 0.01 , suggesting that the scalar invariance hypothesis is supported. Since the three invariance tests were all satisfied, the latent mean analysis was allowed to be conducted in the next stage.

Table 3

Model fit indices for invariance tests

\begin{tabular}{lccccccccc}
\hline \multicolumn{1}{c}{$\chi^{2}$} & $d f$ & $\chi^{2} / d f$ & $\Delta \chi^{2}$ & $\Delta d f$ & $p$ value & CFI & $\Delta$ CFI & RMSEA \\
\hline Configural invariance & 170.684 & 96 & 1.778 & & & & 0.985 & & 0.033 \\
Metric invariance & 178.914 & 104 & 1.72 & 8.23 & 8 & 0.411 & 0.985 & 0 & 0.031 \\
Scalar invariance & 201.768 & 116 & 1.739 & 22.854 & 12 & 0.029 & 0.982 & 0.003 & 0.032 \\
Variance invariance & 207.262 & 120 & 1.727 & 5.493 & 4 & 0.24 & 0.982 & 0 & 0.031 \\
\hline
\end{tabular}

Note: $\mathrm{N}=735($ male $/ n=389$, female/ $n=346)$

\section{Latent mean comparisons}

After the invariance tests, the latent mean analysis approach was conducted to estimate the differences regarding the Internet-specific epistemic beliefs of the male and female groups. Latent mean analysis was performed by constraining the latent means of the male group to be zero. The male group was regarded as the reference group while comparing the latent means with the female group. Because the latent means of the male group were fixed to zero, the latent means of the female group represent the mean differences between the two groups (Hong et al., 2003). The latent mean estimates were displayed in Table 4, indicating that there exist significant differences with respect to uncertainty, complexity and source dimensions of Internet-specific epistemic beliefs. The latent means of female students' Internet-specific epistemic beliefs are higher than those of the male students by $0.25,0.29$ and 0.30 for uncertainty, complexity and source, respectively.

To compute the effect size in a standardised way, it has been suggested that Cohen's $d$ indices could be calculated (Hong et al., 2003; Jang et al., 2012; Kember \& Leung, 2011). First, the assumption of invariant variance across the male and female groups must be satisfied. In turn, the common standard deviations of the two groups can be used to calculate the Cohen's $d$ indices (Hong et al., 2003). As shown in Table 3, the chi-square difference test and change of CFI between the scalar invariance model and the variance invariance model suggest that the variance invariance is supported. Next, the common standard deviations of the male and female groups were used to compute the Cohen's $d$ indices. The Cohen's $d$ indices presented in Table 4 indicate that the values of effect size for uncertainty $(d=0.28)$, complexity $(d=0.30)$ as well as source $(d=0.23)$ are medium. 
Table 4

Results of difference comparison

\begin{tabular}{lccc}
\hline & $\begin{array}{c}\text { Differences of } \\
\text { latent mean }\end{array}$ & $\begin{array}{c}\text { Standard } \\
\text { deviations }\end{array}$ & Cohen's $d$ \\
\hline Uncertainty & $0.25^{* * *}$ & 0.89 & 0.28 \\
Complexity & $0.29^{* * *}$ & 0.99 & 0.30 \\
Source & $0.30^{* *}$ & 1.30 & 0.23 \\
Justification & -0.01 & 0.83 & -0.02 \\
\hline
\end{tabular}

Note: ${ }^{* *} p<0.01^{* * *} p<0.001$

\section{Discussion}

\section{Gender differences in Internet-based learning}

According to the results of the previous research, the gendered issue of Internet-based learning remains controversial. For example, Selwyn (2007) indicated that e-learning can be regarded as a feminine activity. Nevertheless it has been reported that while learning physics, male students tend to show more adaptability to and preferences for constructivist Internet-based learning environments (Chuang et al., 2008). In this study, gender differences in Internet-specific epistemic beliefs were detected while the learning context was defined as searching academic information for course-related tasks in Internet-based learning environments. As Volman and van Eck (2001) indicated, further studies should explore whether various contexts, tasks and learning environments may alter the gender differences in educational computer use. Therefore, there could be a possibility that the learning contexts and tasks may contribute to the varying results regarding the gendered issue in Internet-based learning.

As shown in Table 4, in contrast with their male counterparts, female college students report stronger conceptions regarding the uncertainty, complexity and incomplete source of Internet-based knowledge. This may imply that female students are careful users of Internet-based information while undertaking their course assignments on the Internet. Selwyn (2007) supposed that online learning is a feminine activity as male students may not make much effort to study, while female students tend to use any means such as online learning to study. Compared with male students, female students revealed greater satisfaction with e-learning subjects (Gonzalez-Gomez et al., 2012). Moreover, Bråten and Strømsø (2006) indicated that females are more likely than males to consider Internet-based technologies as useful and appropriate study tools, and to expect to use them for learning. It was also found that test anxiety may significantly explain a certain amount of female students' achievement in online learning environments (Yukselturk \& Bulut, 2009). In sum, it could be postulated that female students' concerns about their study and academic achievement enable them to be careful learners in Internet-based learning environments.

However, holding epistemic beliefs relating to uncertain, complex and incomplete Internet-based knowledge also indicates that learners are more likely to doubt the Internet-based knowledge. It has been argued that students with sophisticated epistemic beliefs in source and complexity (i.e., viewing knowledge as personal construction and believing knowledge to be complex) do not always have better learning outcomes (Bråten, Strømsø, \& Samuelstuen, 2008). As suggested by Li and Kirkup (2007), female students are more likely to underestimate their ability to use the Internet than their counterparts, thus it was reported that females may have difficulty in information searching tasks on the Internet. It was also shown that male students display more perceived control of the Internet and better Internet efficacy than female students do (Peng et al., 2006). Females are more anxious to use web applications for learning than males (Huang et al., 2013). Therefore it should be of concern that female students' low Internet efficacy may result in their over-careful use of Internet-based knowledge. This in turn may cause them to be hesitant in using the course-related information retrieved from the Internet and thus bring about unsatisfactory learning outcomes. From another viewpoint, the relatively more hesitant beliefs in Internet-based knowledge of female students may, to some extent, explain why they usually report lower Internet self-efficacy than males do (Peng et al., 2006; Wu \& Tsai, 2006). 
Peng et al. (2006) also suggested that there is a need to narrow the Internet gender gap by enhancing female students' positive perceptions of the Internet and their Internet self-efficacy. It has been claimed that increasing Internet experience and training programs may be useful in improving students' attitudes and self-efficacy toward the Internet (Wu \& Tsai, 2006). In addition, a well-established constructivist Internetbased learning environment has been recommended to support both males and females' learning on the Internet (Chuang et al., 2008). Hopefully, raising Internet self-efficacy and supported Internet-based environments may eliminate the gender gap in Internet-specific epistemic beliefs and Internet-based learning.

According to Selwyn's (2007) survey, the most masculine aspect of computing application was playing computer games; on the contrary, the more feminine aspect of computing was studying online. Since the online learning has been considered a more feminine activity and favoured by females, to let both genders experience the benefits of e-learning, it was proposed that more masculine-friendly e-learning should be developed to increase males' engagement in Internet-based learning activities (Selwyn, 2007). For male students, it was suggested that the constructivist Internet-based learning environments with challenging problems, cognitive apprenticeship and experts' guidance may attract their preferences toward Internet learning and inspire their epistemic reflection (Tsai, 2008). Regarding the female students, enlarging Internet experience may be a probable way to promote their preferences toward Internet-based learning and self-efficacy which could raise their attitude to the Internet (Durndell \& Haag, 2002; Tsai, 2008).

\section{Gender differences in epistemic beliefs}

Pintrich's (2002) proposition indicated that there are no gender differences in epistemic beliefs when the constructs of epistemology are defined and measured in terms of separate specific dimensions. In opposition to this claim, the findings of this study indicated that gender differences exist in college students' epistemic beliefs in Internet-based learning environments. Elsewhere it has been reported that gender differences exist in epistemic beliefs, but the effect size was generally indicated with a small degree (Kessels, 2013). The results presented in Table 4 display gender differences with a medium effect size in epistemic beliefs regarding Internet-related knowledge. As Mason et al. (2011) indicated that epistemic beliefs are contextsensitive constructs therefore epistemic beliefs may be activated differently in different contexts, that is to say, gender differences regarding epistemic beliefs may also present diversely in Internet-based rather than in traditional learning environments.

Another interesting finding in this study was that no gender gap in justification of Internet-based knowing was found. This implies that male and female students may have different concepts regarding the nature and contents of Internet-based knowledge, however both genders may hold similar beliefs that there is a need to justify the information retrieved from the Internet. Perhaps it can be supposed that popularising access to the Internet in higher education (Wasserman \& Richmond-Abbott, 2005) may result in the fact that both males and females have similar experiences in justifying Internet-based information, and then they may express a similar level of perception about justification of knowing on the Internet.

\section{Suggestions for future research}

Hofer and Pintrich (1997) argued that there is a need to explore the gender-related patterns of epistemic theories; moreover, within-group variability should be examined to understand why there may be gender differences in epistemic theories. Also, it was claimed that the gender differences or online stereotypes may not disappear unless the causes are better understood (Sherman et al., 2000); gender could be conceptualised as contexts (e.g., providing different opportunities, affordances and constraints on individual's development) which need to be detected to evaluate their effects on gender differences in epistemic beliefs (Hofer \& Pintrich, 1997). It was supposed that social developmental differences, social stereotypes and socialisation patterns of genders may account for the digital divide, especially such social expectations may be reinforced by certain contexts (e.g., social contexts such as gender composition of learning groups, learning soft and courses developed by instructors, and gender-specific attribution pattern for the cause of success and failure) which allow the digital divide to continue (Cooper, 2006). Therefore further studies should investigate the causes and contexts which may result in the gender gap regarding epistemic beliefs in Internet-based learning environments. 
It has been claimed that students' epistemic beliefs may predict their Internet-based learning activities including Internet-search and Internet-communication, moreover it was reported that males are more likely to consider the Internet a tool for communication (Bråten \& Strømsø, 2006). In addition, students' perception of the Internet (e.g., perceiving the Internet as toy) may be correlated to their attitudes toward the Internet. It was indicated that male students report more positive attitudes toward the Internet than their female counterparts. Students perceive the Internet as a leisure tool show more positive attitudes than those use the Internet as a functional technology (Peng et al., 2006). Students’ perceptions regarding the nature of the Internet may guide their epistemic beliefs regarding how to use the Internet and interact with the Internet-based environment. In other words, it could be postulated that genders' perceptions of the Internet use which may interplay with epistemic beliefs of genders in Internet-based learning contexts. Further research may explore the role of genders' perception of Internet use in their Internet-specific epistemic beliefs.

In addition to the differences in epistemic beliefs across genders being examined, the cultural effects on epistemic beliefs have also been discussed by previous research (Chai et al., 2012; Rizk et al., 2012). Rizk et al. (2012) further suggested that culture may vary the impacts of gender on epistemic beliefs. For example, the study of Karabenick and Moosa (2005) revealed no gender differences among a Western country (United States) students' epistemic beliefs referring to knowledge from authorities, whereas in a Middle Eastern country -(Oman), gender differences in such epistemic beliefs were found. Future research can examine the gender differences in Internet-specific epistemic beliefs across diverse cultures.

\section{Conclusions}

This study pioneered the execution of latent mean analysis to test the gender differences in Internet-specific epistemic beliefs between male and female students while searching for course-related information on the Internet. Gender differences no longer exist in computer ownership of young adults in university (Li \& Kirkup, 2007). However it has been claimed that even though Internet access and exposure have improved, the Internet gender gap has not diminished but still exists in Internet use (Jones et al., 2009). In other words, being aware of the gender differences in Internet activities and conceptions of Internet-specific knowledge, instructors and educators can develop relevant Internet-based learning environments and curricula to motivate both male and female students to become involved in such learning.

\section{Acknowledgements}

This study was supported by the Ministry of Science and Technology, Taiwan, under grant numbers MOST 101-2511-S-011-003-MY3, MOST 101-2628-S-011-001-MY3, MOST 103-2511-S-011-004-MY2 and MOST 103-2511-S-011-008-MY3.

\section{References}

Bandalos, D. L., \& Finney, S. J. (2010). Factor analysis: Exploratory and confirmatory. In G. R. Hancock, \& R. O. Mueller (Eds.), Quantitative methods in the social and behavioral sciences: A guide for researchers and reviewers (pp. 93-114). New York, NY: Routledge.

Bendixen, L. D., Schraw, G., \& Dunkle, M. E. (1998). Epistemic beliefs and moral reasoning. Journal of Psychology, 132(2), 187-200. doi.org/10.1080/00223989809599158

Biddix, J. P., Chung, C. J., \& Park, H. W. (2011). Convenience or credibility? A study of college student online research behaviors. The Internet and Higher Education, 14(3), 175-182. doi.org/10.1016/j.iheduc.2011.01.003

Bråten, I., \& Strømsø, H. I. (2006). Epistemological beliefs, interest, and gender as predictors of Internetbased learning activities. Computers in Human Behavior, 22(6), 1027-1042. doi.org/10.1016/j.chb.2004.03.026

Bråten, I., Strømsø, H. I., \& Samuelstuen, M. S. (2005). The relationship between Internet-specific epistemological beliefs and learning within Internet technologies. Journal of Educational Computing Research, 33(2), 141-171. doi.org/10.2190/E763-X0LN-6NMF-CB86

Bråten, I., Strømsø, H. I., \& Samuelstuen, M. S. (2008). Are sophisticated students always better? The role of topic-specific personal epistemology in the understanding of multiple expository texts. Contemporary Educational Psychology, 33(4), 814-840. doi.org/10.1016/j.cedpsych.2008.02.001 
Byrne, B. M. (2010). Structural equation modeling with AMOS: Basic concepts, applications, and programming (2nd ed.). New York, NY: Routledge.

Chai, C. S., Deng, F., \& Tsai, C. C. (2012). A comparison of scientific epistemological views between mainland China and Taiwan high school students. Asia Pacific Education Review, 13(1), 17-26. doi.org/10.1007/s12564-011-9174-9

Chen, F. F. (2007). Sensitivity of goodness of fit indexes to lack of measurement invariance. Structural Equation Modeling: A Multidisciplinary Journal, 14(3), 464-504. doi.org/10.1080/10705510701301834

Chen, R. S., \& Tsai, C. C. (2007). Gender differences in Taiwan university students' attitudes toward webbased learning. CyberPsychology \& Behavior, 10(5), 645-654. doi.org/10.1089/cpb.2007.9974

Cheung, G. W., \& Rensvold, R. B. (2002). Evaluating goodness-of-fit indexes for testing measurement invariance. Structural Equation Modeling: A Multidisciplinary Journal, 9(2), 233-255. doi.org/10.1207/S15328007sem0902_5

Chiu, Y.-L., Liang, J.-C., \& Tsai, C.-C. (2013). Internet-specific epistemic beliefs and self-regulated learning in online academic information searching. Metacognition and Learning. doi.org/10.1007/s11409-013-9103-X

Chou, C., Wu, H. C., \& Chen, C. H. (2011). Re-visiting college students' attitudes toward the Internetbased on a 6-T model: Gender and grade level difference. Computers \& Education, 56(4), 939-947. doi.org/10.1016/j.compedu.2010.11.004

Chuang, S. C., Hwang, F. K., \& Tsai, C. C. (2008). Students' perceptions of constructivist internet learning environments by a physics virtual laboratory: The gap between ideal and reality and gender differences. Cyberpsychology \& Behavior, 11(2), 150-156. doi.org/10.1089/cpb.2007.0024

Conley, A. M., Pintrich, P. R., Vekiri, L., \& Harrison, D. (2004). Changes in epistemological beliefs in elementary science students. Contemporary Educational Psychology, 29(2), 186-204. doi.org/10.1016/j.cedpsych.2004.01.004

Cooper, J. (2006). The digital divide: the special case of gender. Journal of Computer Assisted Learning, 22(5), 320-334. doi.org/10.1111/j.1365-2729.2006.00185.x

Dholakia, R. R. (2006). Gender and IT in the household: Evolving patterns of Internet use in the United States. Information Society, 22(4), 231-240. doi.org/10.1080/01972240600791374

Durndell, A., \& Haag, Z. (2002). Computer self efficacy, computer anxiety, attitudes towards the Internet and reported experience with the Internet, by gender, in an East European sample. Computers in Human Behavior, 18(5), 521-535. doi.org/10.1016/S0747-5632(02)00006-7

Gonzalez-Gomez, F., Guardiola, J., Rodriguez, O. M., \& Alonso, M. A. M. (2012). Gender differences in e-learning satisfaction. Computers \& Education, 58(1), 283-290. doi.org/10.1016/j.compedu.2011.08.017

Gregorich, S. E. (2006). Do self-report instruments allow meaningful comparisons across diverse population groups? Testing measurement invariance using the confirmatory factor analysis framework. Medical Care, 44(11), S78-S94. doi.org/10.1097/01.mlr.0000245454.12228.8f

Hofer, B. K., \& Pintrich, P. R. (1997). The development of epistemological theories: beliefs about knowledge and knowing and their relation to learning. Review of Educational Research, 67(1), 88140. doi.org/10.3102/00346543067001088

Hong, S., Malik, M. L., \& Lee, M. K. (2003). Testing configural, metric, scalar, and latent mean invariance across genders in sociotropy and autonomy using a non-western sample. Educational and Psychological Measurement, 63(4), 636-654. doi.org/10.1177/0013164403251332

Huang, W. H. D., Hood, D. W., \& Yoo, S. J. (2013). Gender divide and acceptance of collaborative Web 2.0 applications for learning in higher education. The Internet and Higher Education, 16, 57-65. doi.org/10.1016/j.iheduc.2012.02.001

Jang, Y. J., Lee, J., Puig, A., \& Lee, S. M. (2012). Factorial invariance and latent mean differences for the five factor wellness inventory with Korean and American counselors. Measurement and Evaluation in Counseling and Development, 45(2), 71-83. doi.org/10.1177/0748175611427915

Jones, S., Johnson-Yale, C., Millermaier, S., \& Perez, F. S. (2009). US college students' Internet use: Race, gender and digital divides. Journal of Computer-Mediated Communication, 14(2), 244-264. doi.org/10.1111/j.1083-6101.2009.01439.x

Karabenick, S. A., \& Moosa, S. (2005). Culture and personal epistemology: U.S. and Middle Eastern students' beliefs about scientific knowledge and knowing. Social Psychology of Education, 8(4), 375393. doi.org/ 10.1007/s11218-005-1826-3

Kember, D., \& Leung, D. Y. P. (2011). Disciplinary differences in student ratings of teaching quality. Research in Higher Education, 52(3), 278-299. doi.org/10.1007/s11162-010-9194-z 
Kessels, U. (2013). How epistemological beliefs relate to values and gender orientation. Learning and Individual Differences, 23, 256-261. doi.org/10.1016/j.lindif.2012.10.008

Kline, R. B. (2005). Principles and practice of structural equation modeling. New York, NY: Guilford Press.

Kuhn, J. T., \& Holling, H. (2009). Measurement invariance of divergent thinking across gender, age, and school forms. European Journal of Psychological Assessment, 25(1), 1-7. doi.org/10.1027/10155759.25.1.1

Li, N., \& Kirkup, G. (2007). Gender and cultural differences in Internet use: A study of China and the UK. Computers \& Education, 48(2), 301-317. doi.org/10.1016/j.compedu.2005.01.007

Liang, J. C., \& Tsai, C. C. (2009). The information commitments toward web information among medical students in Taiwan. Educational Technology \& Society, 12(1), 162-172.

Mason, L., Ariasi, N., \& Boldrin, A. (2011). Epistemic beliefs in action: Spontaneous reflections about knowledge and knowing during online information searching and their influence on learning. Learning and Instruction, 21, 137-151. doi.org/10.1016/j.learninstruc.2010.01.001

Mason, L., \& Boldrin, A. (2008). Epistemic metacognition in the context of information searching on the Web. In M. S. Khine (Ed.), Knowing, knowledge and beliefs: Epistemological studies across diverse cultures (pp. 377-404). New York, NY: Springer.

Metzger, M. J., Flanagin, A. J., \& Zwarun, L. (2003). College student Web use, perceptions of information credibility, and verification behavior. Computers \& Education, 41(3), 271-290. doi.org/10.1016/S0360-1315(03)00049-6

Ong, C. S., \& Lai, J. Y. (2006). Gender differences in perceptions and relationships among dominants of e-learning acceptance. Computers in Human Behavior, 22(5), 816-829. doi.org/10.1016/j.chb.2004.03.006

Peng, H. Y., Tsai, C. C., \& Wu, Y. T. (2006). University students' self-efficacy and their attitudes toward the Internet: the role of students' perceptions of the Internet. Educational Studies, 32(1), 73-86. doi.org/10.1080/03055690500416025

Pintrich, P. R. (2002). Future challenges and directions for theory and research on personal epistemology. In B. K. Hofer, \& P. R. Pintrich (Eds.), Personal epistemology: The psychology of beliefs about knowledge and knowing (pp. 389-414). Mahwah, NJ: Lawrence Erlbaum Associates.

Rizk, N., Jaber, L., Halwany, S., \& BouJaoude, S. (2012). Epistemological beliefs in science: An exploratory study of Lebanese university students' epistemologies. International Journal of Science and Mathematics Education, 10(3), 473-496. doi.org/10.1007/s10763-011-9289-9

Schommer, M. (1990). Effects of beliefs about the nature of knowledge on comprehension. Journal of Educational Psychology, 82(3), 498-504. doi.org/10.1037//0022-0663.82.3.498

Selwyn, N. (2007). e-Learning or she-learning? Exploring students' gendered perceptions of education technology. British Journal of Educational Technology, 38(4), 744-746. doi.org/10.1111/j.14678535.2006.00659.x

Shaw, L., \& Gant, L. (2002). Users divided? Exploring the gender gap in Internet use CyberPsychology \& Behavior, 5(6), 517-527. doi.org/10.1089/109493102321018150

Sherman, R. C., End, C., Kraan, E., Cole, A., Campbell, J., Birchmeier, Z., \& Klausner, J. (2000). The Internet gender gap among college students: Forgotten but not gone? Cyberpsychology \& Behavior, 3(5), 885-894. doi.org/10.1089/10949310050191854

Strømsø, H. I., \& Bråten, I. (2010). The role of personal epistemology in the self-regulation of internetbased learning. Metacognition and Learning, 5(1), 91-111. doi.org/10.1007/s11409-009-9043-7

Tsai, C. C. (2004). Beyond cognitive and metacognitive tools: The use of the Internet as an 'epistemological' tool for instruction. British Journal of Educational Technology, 35(5), 525-536. doi.org/10.1111/j.0007-1013.2004.00411.x

Tsai, C. C. (2006). Biological knowledge is more tentative than physics knowledge: Taiwan high school adolescents' views about the nature of biology and physics. Adolescence, 41(164), 691-703.

Tsai, C. C. (2008). The preferences toward constructivist Internet-based learning environments among university students in Taiwan. Computers in Human Behavior, 24(1), 16-31. doi.org/10.1016/j.chb.2006.12.002

Tsai, C. C., \& Chuang, S. C. (2005). The correlation between epistemological beliefs and preferences toward Internet-based learning environments. British Journal of Educational Technology, 36(1), $97-$ 100. doi.org/10.1111/j.1467-8535.2004.00442.x

Tsai, M. J., Liang, J. C., Hou, H. T., \& Tsai, C. C. (2012). University students' online information searching strategies in different search contexts. Australasian Journal of Educational Technology, 28(5), 881-895. Retrieved from http://www.ascilite.org.au/ajet/ajet28/tsai-mj.html 
Tsai, M. J., \& Tsai, C. C. (2010). Junior high school students' Internet usage and self-efficacy: A reexamination of the gender gap. Computers \& Education, 54(4), 1182-1192. doi.org/10.1016/j.compedu.2009.11.004

Volman, M., \& van Eck, E. (2001). Gender equity and information technology in education: The second decade. Review of Educational Research, 71(4), 613-634. doi.org/10.3102/00346543071004613

Wasserman, I. M., \& Richmond-Abbott, M. (2005). Gender and the Internet: Causes of variation in access, level, and scope of use. Social Science Quarterly, 86(1), 252-270. doi.org/10.1111/j.00384941.2005.00301.x

Wong, K. T., Teo, T., \& Russo, S. (2012). Influence of gender and computer teaching efficacy on computer acceptance among Malaysian student teachers: An extended technology acceptance model. Australasian Journal of Educational Technology, 28(7), 1190-1207. Retrieved from http://www.ascilite.org.au/ajet/ajet28/wong-kt.html

Wu, Y. T., \& Tsai, C. C. (2006). University students' Internet attitudes and Internet self-efficacy: A study at three universities in Taiwan. CyberPsychology \& Behavior, 9(4), 441-450. doi.org/10.1089/cpb.2006.9.441.

Yang, F. Y., \& Chang, C. C. (2009). Examining high-school students' preferences toward learning environments, personal beliefs and concept learning in web-based contexts. Computers \& Education, 52, 848-857. doi.org/10.1016/j.compedu.2008.12.008

Yukselturk, E., \& Bulut, S. (2009). Gender differences in self-regulated online learning environment. Educational Technology \& Society, 12(3), 12-22.

Corresponding author: Jyh-Chong Liang, aljc@mail.ntust.edu.tw

Australasian Journal of Educational Technology (C) 2015.

Please cite as: Chiu, Y-L., Tsai, C-C. \& Liang, J-C. (2015). Testing measurement invariance and latent mean differences across gender groups in college students' Internet-specific epistemic beliefs. Australasian Journal of Educational Technology, 31(4), 486-499. 